\title{
In Vitro and In Vivo Operation of a Wireless Body Sensor Node
}

\author{
Francesco Merli ${ }^{1}$, Léandre Bolomey ${ }^{2}$, François Gorostidi ${ }^{3}$, Yann Barrandon ${ }^{3}$, \\ Eric Meurville ${ }^{2}$, and Anja K. Skrivervik ${ }^{1}$ \\ ${ }^{1}$ Laboratory of Electromagnetics and Acoustics, \\ 2 Laboratory of Microengineering for Manufacturing 2, \\ Ecole Polytechnique Fédérale de Lausanne, \\ 3 Laboratory of Stem Cell Dynamics, \\ Ecole Polytechnique Fédérale de Lausanne and Department of Experimental Surgery, \\ Centre Hospitalier Universitaire Vaudois and University of Lausanne \\ Switzerland \\ \{francesco.merli, eric.meurville, anja.skrivervik\}@epfl.ch, \\ \{francois.gorostidi, yann.barrandon\}@epfl.ch, \\ leandre.bolomey@a3.epfl.ch
}

\begin{abstract}
Summary. A wireless Body Sensor Node (BSN) and its operations are presented. The BSN comprises all the necessary components (i.e., antenna, electronics, batteries and bio-sensor) to allow continuous monitoring of physiological data. In vitro characterization validates the simulated performances, while in vivo experiment shows the capability of the system for real life telemedicine applications.
\end{abstract}

Key words: Body Sensor Node (BSN), implantable antennas, implantable telemetry system, Medical Device Radiocommunications Service (MedRadio), Telemedicine.

\section{Introduction}

Wireless implantable systems promise large improvements in patients' care and quality of life. For this purpose, small biocompatible devices have been recently presented for different applications such as $\mathrm{pH}$ monitoring [1], gastro-intestinal tract exploration $[2,3]$ or cardiovascular pressure control [4]. In this work we present a complete Body Sensor Node which has been tested in vitro and applied in vivo in a porcine animal for local temperature monitoring. The system performs data telemetry with an external Base Station in the Medical Device Radiocommunications Service band (MedRadio, 401-406 MHz) [5] and the Industrial, Scientific and Medical band (ISM, 2.4-2.5 GHz).

\section{Body Sensor Node}

The proposed BSN aims at a high system integration of all its components, namely: the Multilayered Spiral Antenna (MSA), the electronics (the RF transceiver 
and the Digital Signal Processor), the batteries and the bio-sensor. Fig. 1 depicts the complete packaging of the device. All the elements fit in a biocompatible cylindrical housing which measures $10 \times 32[\mathrm{~mm}]$.

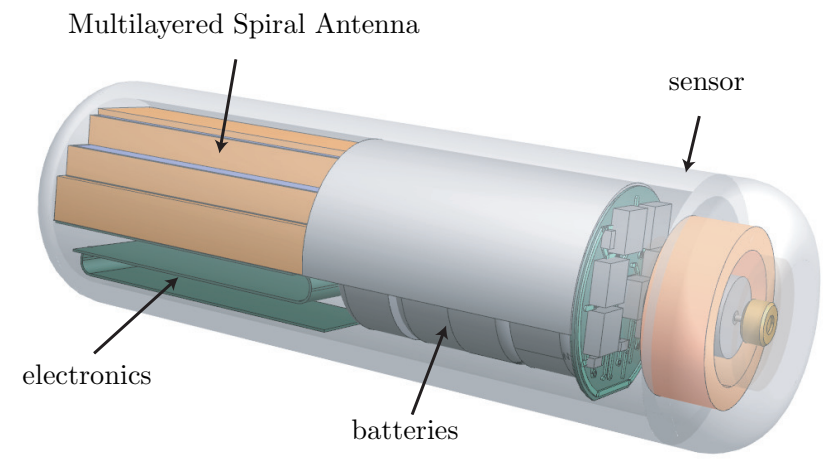

Fig. 1: Complete packaging of the proposed BSN. The biocompatible casing is in light gray. As a possible sensor, the driver and rotor for glucose microviscometer described in [6] are illustrated.

The MSA consists in a conformal radiator whose main resonating element is a three dimensional spiral metallization developed on a pyramidal assembly as described in $[7,8]$. Polyetheretherketones (PEEK) was used for the biocompatible housing, as it is very easy to machine and its biocompatible characteristics are well established $[9,10]$. The housing thickness $(0.8 \mathrm{~mm})$ was selected to improve the electromagnetic radiation of the implanted radiator in agreement with the results reported in [11]. The MSA has dual band capabilities working in both the MedRadio and the ISM bands and its radiation performances (maximum gain equal to -29.4 and $-17.7 \mathrm{dBi}$ in the lower and higher frequency ranges, respectively) provide a robust communication link for applications targeting a minimum working range of $2 \mathrm{~m}$.

The electronics components were assembled on a flexible Printed Circuit Board (PCB) [12] to fit in the small available volume. The RF communication is provided by an ultra-low power Integrated Circuit (IC), the ZL70101 [13] manufactured by Zarlink, operating in both the MedRadio and ISM bands. The BSN operations are executed by an ultra-low power digital signal processor: the Ezairo 5900 manufactured by ON Semiconductor. Four coin type 377 batteries $(1.5 \mathrm{~V} / 27 \mathrm{mAh})$, manufactured by Energizer, were selected to provide the required power supply.

The conception of the proposed BSN gives a broad freedom regarding the monitoring device or bio-actuator to be included. The front-end electronics to drive the glucose microviscometer presented in $[6]$ and the potentionstat described in [14] are just examples of possible sensors. 


\section{Power Consumption}

In order to reduce the power requirements and to extend the life time of the BSN, the system is kept in a sleeping state. In this condition only the $2.45 \mathrm{GHz}$ wake-up part of the transceiver is active and the power consumption is $2 \mu \mathrm{W}$. A signal received in the ISM band wakes up the IC; subsequently, the measurements are performed and the bidirectional communication occurs in the MedRadio frequency spectrum. Considering the TMP112 temperature sensor from Texas Instruments (used in the in vivo experiment), the total active phase lasts $430 \mathrm{~ms}$ and consumes $15.7 \mathrm{~mW}$. If one measurement is taken every 5 minutes, the embedded batteries provide a life time of 284 days. The power ratio of each part of the BSN is depicted in Fig. 6 (RF 91.9\%, DSP 8.0\%, sensor 0.1\%). The RF portion can be separated in the actual power used to transmit data $\left(\mathrm{RF}_{\mathrm{TX}}=2.7 \%\right)$, to establish the MedRadio communication channel $\left(\mathrm{RF}_{\text {est }}=14.6 \%\right)$ and to reserve the channel until the data are ready to be sent $\left(\mathrm{RF}_{\mathrm{ch}}=74.6 \%\right)$, as illustrated in Fig. 2 .

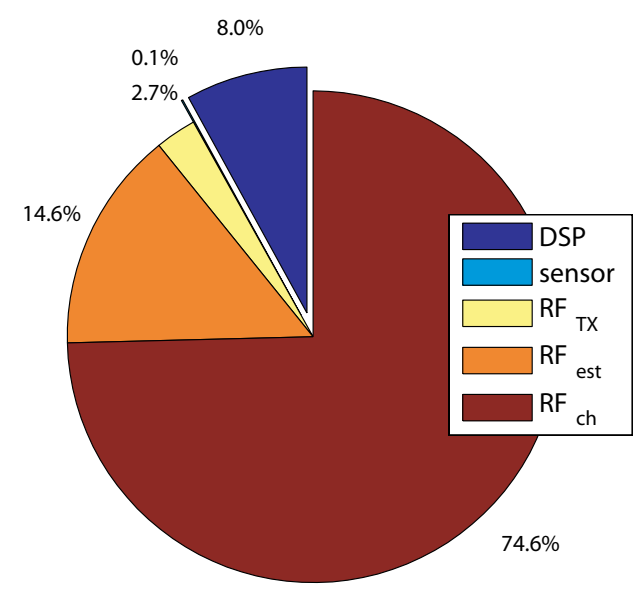

Fig. 2: Power consumption repartition among the different components of the BSN during the active phase.

Comparing the performances of implantable devices with telemetry capabilities is not an easy task as many different conditions (working frequency, data rate, duty cycle dimension, implant location, purpose, power supply) can be considered. In Fig. 3 one can notice the good compromise of the proposed BSN 
among volume occupation, power consumption and life time when compared to other implantable systems ${ }^{1}$.

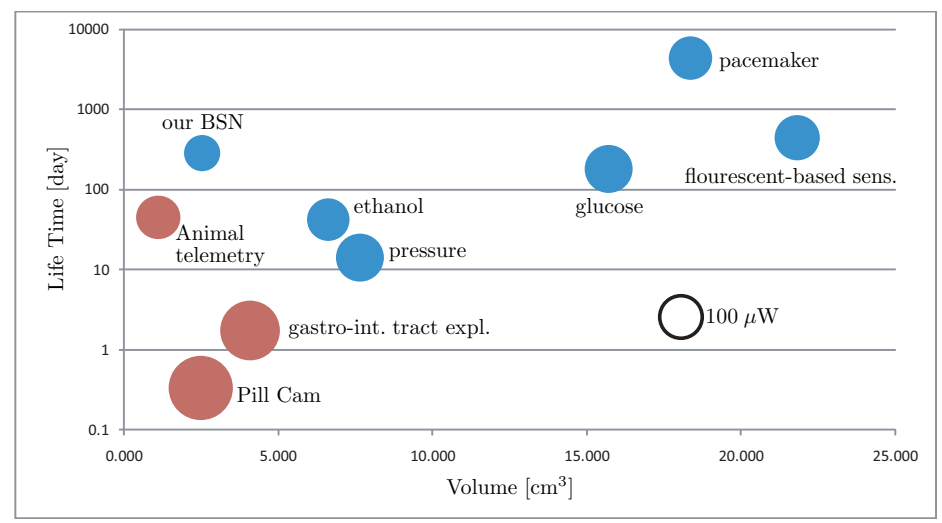

Fig. 3: Graphical comparison of active implantable devices with far field (blue) and near field (red) communication capability: flourescent-based sens. [15], pacemaker [16], glucose [17], pressure [18], ethanol [19], gastro-int. tract expl. [3], Pill Cam [2], Animal telemetry [20] and our BSN. The size of the bubble reflects indicated the power consumption computed as the ration between life time and the battery supplies.

\section{In Vitro Characterization}

In vitro tests were performed in order to check the functioning of the realized device and validate the MSA radiation characteristic. The BSN was inserted in a liquid body phantom. The latter has dielectric properties equivalent to the human muscle tissue and has a cylindrical shape $(80 \times 110[\mathrm{~mm}])$. Outdoor test were carried out to assess the capability of the MedRadio communication link (as illustrated in Fig. 4-(a)), while the wake-up performances in the $2.45 \mathrm{GHz}$ ISM band were verified in an anechoic chamber.

Maximum working ranges, reported in Fig. 4-(b), confirmed the simulated performances of the MSA. Considering the power link budget characteristics of the IC ZL70101 and the Base Station provided by Zarlink, the maximum registered ranges correspond to antenna gain values equal to -30.5 and $-18.6 \mathrm{dBi}$ in the MedRadio and ISM bands, respectively. These values, which take into account the mismatch and the losses within the electronics assembly, closely agree with the predicted characteristics, i.e., -29.4 and $-17.7 \mathrm{dBi}$.

\footnotetext{
${ }^{1}$ The selected implantable sensors are only chosen to show examples of different applications as the list is by no means exhaustive.
} 


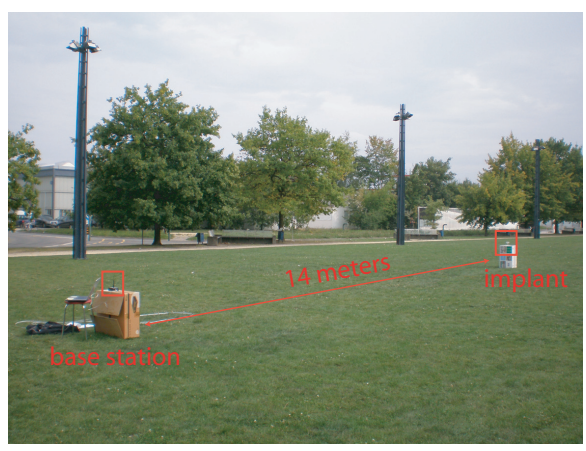

\begin{tabular}{l|c}
\hline \hline Frequency & Range $[\mathbf{m}]$ \\
\hline \hline $403 \mathrm{MHz}$ & 14.0 \\
\hline $2.47 \mathrm{GHz}$ & 4.8 \\
\hline \hline
\end{tabular}

(b)

(a)

Fig. 4: In vitro characterization: (a) outdoor communication tests and (b) maximum registered ranges for both working frequencies.

\section{In Vivo Experiment}

Two BSNs were implanted in a large animal model (Göttingen minipigs) chosen for the similarity between their and the human tissues. A temperature sensor was included into the BSNs to study the correlation between local temperature and the healing process of a deep wound in the settings of a cultured epidermal autograft. In fact, it has been observed that the temperature has an important impact on epidermal stem cell behavior in vitro [21].

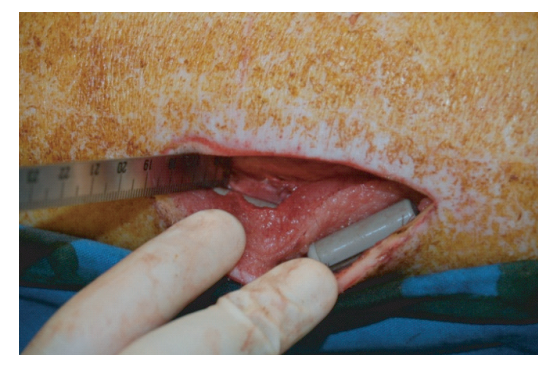

Fig. 5: Implantation, in accordance to all ethical considerations and the regulatory issues related to animal experiments, of the two BSNs at different depths. A subcutaneous location ( $5 \mathrm{~mm}$ deep) and an intra-muscular one (30 $\mathrm{mm}$ deep), were chosen.

In vivo experiment lasted for 15 consecutive days. During this period the animal dwelled most of the time in a farm and periodically, it was taken away for a follow-up at the hospital. Fig. 6 shows the registered temperatures during one of these follow-ups. Values measured by a rectal probe are also reported to appreciate the effect of the placement of the two BSNs. 


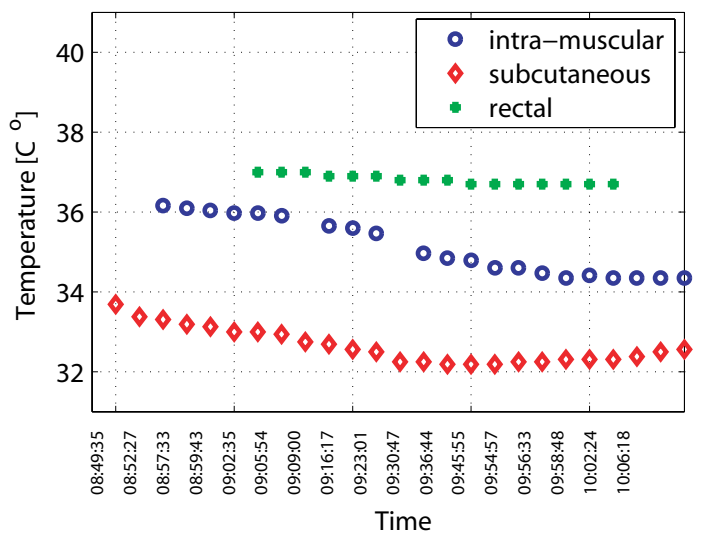

Fig. 6: Measured temperature values at the hospital.

While being at the farm, the animal was maintained indoor in a cage of dimensions $1.3 \times 2.7[\mathrm{~m}]$. In order not to interfere with the farm daily life (feeding of the animals, cleaning, etc.), the Base Station was placed in the mansard above the cage room at a distance of $2.5 \mathrm{~m}$.

The overall performances during the entire test period are reported in Table 1. During the 2696 interrogations between each BSN and the external Base Station the relative number of failed communications are $5.82 \%$ and $16.10 \%$ for the subcutaneous and intra-muscular BSN, respectively. From the point of view of communication protocol, three sources of error are identified in Table 1: MedRadio, ISM and firmware. One can appreciate that the data transmission in the MedRadio was found to be the most critical on for the deepest sensor $\left(\operatorname{err}_{\text {MedRadio }}=12.46 \%\right.$ ), while the wake-up communication showed a relative number of errors err ISM lower than $5 \%$ for both BSNs. Almost negligible problems were caused by the driving firmware $\left(\operatorname{err}_{\text {firmware }}<0.2 \%\right.$ ). Explanations for the registered errors have been found observing the pig activity in the cage and the consequent relative positions between the BSNs and the Base Station.

Table 1: Communication Performances during the In Vivo Experiment.

\begin{tabular}{l|c|c|c|c|c}
\hline \hline BSN & $\begin{array}{c}\text { Number of } \\
\text { Measurements }\end{array}$ & $\begin{array}{c}\operatorname{err}_{\text {MedRadio }} \\
{[\%]}\end{array}$ & $\begin{array}{c}\operatorname{err}_{\text {firmware }} \\
{[\%]}\end{array}$ & $\begin{array}{c}\operatorname{err}_{\text {ISM }} \\
{[\%]}\end{array}$ & $\begin{array}{c}\operatorname{err}_{\text {tot }} \\
{[\%]}\end{array}$ \\
\hline \hline intra-muscular & 2696 & 12.46 & 0.00 & 3.63 & 16.09 \\
\hline subcutaneous & 2696 & 1.67 & 0.11 & 4.04 & 5.82 \\
\hline \hline
\end{tabular}




\section{Conclusion}

This work presented the operation of a complete wireless Body Sensor Node. The node integrates all the necessary components in a cylindrical volume $(10 \times 32[\mathrm{~mm}])$. Communication with an external Base Station placed in a few meters working range (up to $14 \mathrm{~m}$ ) has been proven. The results obtained by the in vitro and in vivo tests confirm the promising capabilities of the proposed BSN and pave the way for future research oriented to the making of complete telemedicine systems.

\section{Acknowlegments}

The authors would like to thank J.-F. Zürcher and G. Corradini for the help provided during the realization of the proposed BSN.

\section{References}

[1] J. L. Gonzalez-Guillaumin, D. C. Sadowski, K. V. I. S. Kaler, and M. P. Mintchev, "Ingestible capsule for impedance and $\mathrm{pH}$ monitoring in the esophagus,", IEEE Trans. Biomed. Eng., vol. 54, no. 12, pp. 2231-2236, Dec. 2007.

[2] Pill cam, Given Imaging. [Online]. Available: http://www.givenimaging. com/en-us/Pages/GivenWelcomePage.aspx

[3] E. A. Johannessen, L. Wang, C. Wyse, D. R. S. Cumming, and J. M. Cooper, "Biocompatibility of a lab-on-a-pill sensor in artificial gastrointestinal environments," IEEE Trans. Biomed. Eng., vol. 53, no. 11, pp. 2333-2340, Nov. 2006.

[4] E. Y. Chow, A. L. Chlebowski, S. Chakraborty, W. J. Chappell, and P. P. Irazoqui, "Fully wireless implantable cardiovascular pressure monitor integrated with a medical stent," IEEE Trans. Biomed. Eng., vol. 57, no. 6, pp. 1487-1496, 2010.

[5] Medical Device Radiocommunications Service (MedRadio), Federal Communication Commission (FCC) Std. CFR, Part 95.601-673 Subpart E, Part 95.1201-1221 Subpart I, 2009, formerly Medical Implanted Communication System (MICS). [Online]. Available: http://wireless.fcc.gov/services/index. htm?job=service $\backslash$ _home $\backslash$ \&id $=$ medical $\backslash$ _implant

[6] S. Kuenzi, E. Meurville, and P. Ryser, "Automated characterization of dextran/concanavalin a mixtures-a study of sensitivity and temperature dependence at low viscosity as basis for an implantable glucose sensor," Sensors and Actuators B: Chemical, vol. 146, no. 1, pp. 1-7, 2010.

[7] F. Merli, L. Bolomey, E. Meurville, and A. K. Skrivervik, "Dual band antenna for subcutaneous telemetry applications," in Proc. IEEE Antennas and Propagation Society Int. Symp. (APSURSI), 2010, pp. 1-4. 
[8] F. Merli, L. Bolomey, J.-F. Zürcher, G. Corradini, E. Meurville, and A. K. Skrivervik, "Design, realization and measurements of a miniature antenna for implantable wireless communication systems," IEEE Trans. Antennas Propag., accepted for publication.

[9] E. A. Johannessen, L. Wang, L. Cui, T. B. Tang, M. Ahmadian, A. Astaras, S. W. J. Reid, P. S. Yam, A. F. Murray, B. W. Flynn, S. P. Beaumont, D. R. S. Cumming, and J. M. Cooper, "Implementation of multichannel sensors for remote biomedical measurements in a microsystems format," IEEE Trans. Biomed. Eng., vol. 51, no. 3, pp. 525-535, Mar. 2004.

[10] S. M. Kurtz and J. N. Devine, "PEEK biomaterials in trauma, orthopedic, and spinal implants," Biomaterials, vol. 28, no. 32, pp. 4845-4869, Nov. 2007.

[11] F. Merli, B. Fuchs, J. R. Mosig, and A. K. Skrivervik, "The effect of insulating layers on the performance of implanted antennas," IEEE Trans. Antennas Propag., vol. 59, no. 1, pp. 21 -31, Jan. 2011.

[12] L. Bolomey, E. Meurville, and P. Ryser, "Implantable ultra-low power DSPbased system for a miniature chemico-rheological biosensor," in Proceedings of the Eurosensors XXIII conference, no. 1, 2009, pp. 1235 - 1238.

[13] P. D. Bradley, "Implantable ultralow-power radio chip facilitates in-body communications," in RF Design (online magazine), 2007. [Online]. Available: http://rfdesign.com/next\_generation\_wireless/short _range $\backslash$-wireless $/ 706\{$ RFDF $\} 1$.pdf

[14] G. De Micheli, S. Ghoreishizadeh, C. Boero, F. Valgimigli, and S. Carrara, "An integrated platform for advanced diagnostics," in Proc. Design, Automation and Test in Europe (DATE 2011), 2011, pp. 2995-2999.

[15] P. Valdastri, E. Susilo, T. Forster, C. Strohhofer, A. Menciassi, and P. Dario, "Wireless implantable electronic platform for chronic fluorescent-based biosensors," IEEE Trans. Biomed. Eng., vol. 58, no. 6, pp. 1846-1854, 2011.

[16] S. Audet, E. J. Herrmann, R. Receveur et al., "Medical applications," Comprehensive Microsystems, pp. 421-474, 2008.

[17] P. Valdastri, E. Susilo, T. Förster, C. Strohhöfer, A. Menciassi, and P. Dario, "Wireless implantable electronic platform for blood glucose level monitoring," in Proceedings of the Eurosensors XXIII conference, no. 1, 2009, pp. $1255-1258$.

[18] P. Valdastri, A. Menciassi, A. Arena, C. Caccamo, and P. Dario, "An implantable telemetry platform system for in vivo monitoring of physiological parameters," IEEE Trans. Inf. Technol. Biomed., vol. 8, no. 3, pp. 271-278, Sept. 2004.

[19] C. P. Cheney, B. Srijanto, D. L. Hedden, A. Gehl, T. L. Ferrell, J. Schultz, E. A. Engleman, W. J. McBride, and S. O'Connor, "In vivo wireless ethanol vapor detection in the wistar rat." Sens Actuators B Chem, vol. 138, no. 1, pp. 264-269, Apr. 2009.

[20] DSI PhysioTel PA-C10 for Mice, data sciences international 2005.

[21] Brouard and Barrandon, Private Communication. 